\title{
Zusammenfassende Schlussbetrachtung
}

\author{
Horst Gömann und Johanna Fick
}

\section{Inhaltsverzeichnis}

6.1 Änderung von Rahmenbedingungen nach Abschluss der Studie. . . . . . . . . . . . . 422

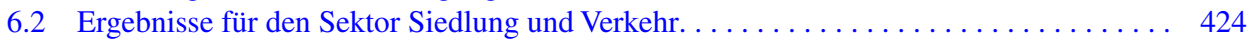

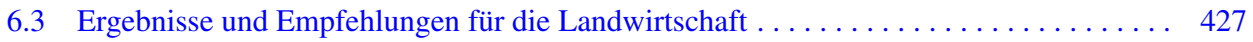

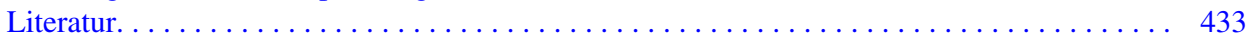

In Deutschland hat die Bedeutung des Themas Klimawandel in der öffentlichen Wahrnehmung aufgrund einer Häufung extremer Wetterlagen - wie Starkregenereignisse, Überflutungen und vor allem die extreme Hitze und Dürre - in den letzten Jahren zugenommen. Im gesellschaftlichen und politischen Diskurs wird der Notwendigkeit, stärker als bisher zum Schutz des Klimas auf eine Minderung von THG-Emissionen hinzuwirken, eine höhere Priorität beigemessen. Im vorliegenden Buch werden ausgewählte Ergebnisse des Verbundvorhabens CC-LandStraD dargestellt, das die komplexen Wechselwirkungen zwischen Klimawandel und Landnutzung sowie Landnutzungsänderungen in den Sektoren „Siedlung und Verkehr“, „Landwirtschaft“" und „Forstwirtschaft" mit Blick auf die Minderung von THG-Emissionen analysierte.

Mit Hilfe eines interdisziplinären Modellverbundes wurden ausgewählte Maßnahmen der Landnutzung sowie Maßnahmenbündel, die im transdisziplinären Diskurs mit Akteuren diskutiert und ausgearbeitet wurden, hinsichtlich ihrer Auswirkungen

H. Gömann ( $₫)$ · J. Fick

Thünen-Institut für Ländliche Räume, Braunschweig, Deutschland

E-Mail: cc-landstrad@thuenen.de

(C) Der/die Autor(en) 2021

H. Gömann und J. Fick (Hrsg.), Wechselwirkungen zwischen Landnutzung und

Klimawandel, https://doi.org/10.1007/978-3-658-18671-5_6 
untersucht, insbesondere mit Blick auf THG-Emissionen. Die Ergebnisse der modellgestützten Maßnahmewirkungsanalysen geben Anhaltspunkte zur Einschätzung der jeweiligen Einsparpotenziale und den damit verbundenen Kosten, die wichtige Faktoren für die Bewertung von Maßnahmen und Maßnahmenbündeln (Strategien) sind. Die Modelle bilden einen Stand der Technologie ab, wobei vielfältige Annahmen getroffen werden mussten, auch zu den Anpassungsmechanismen, die letztlich das Verhalten der abgebildeten Akteure widerspiegeln sollen. Die Maßnahmenbündel, sogenannte Landnutzungsstrategien, fokussierten auf Klimaschutz, Bioenergie, Natur- und Umweltschutz bzw. auf Klimaanpassung. Bei den Maßnahmewirkungsanalysen wurde von konstanten Konsumgewohnheiten ausgegangen, sodass Landnutzungsänderungen, die beispielsweise zu einer Verringerung der Nahrungsproduktion in Deutschland führen, durch entsprechende Im- oder Exporte ausgeglichen werden müssen, die in anderen Regionen der Welt zu THG-Emissionen führen (Indirect Land Use Change: ILUC). Maßnahmen im Verkehrs- oder Gebäudebereich sowie in der Tierhaltung wurden nicht adressiert.

Die Landnutzung erfüllt verschiedene gesellschaftliche Ansprüche und Funktionen, sogenannte Ökosystemfunktionen wie die Erzeugung von Nahrung und Rohstoffen, die Bereitstellung von Raum für Wohnen, Gewerbe sowie Erholung. Darüber hinaus soll die Landnutzung im Einklang mit der Natur und Umwelt erfolgen. Die Integration eines verstärkten Klimaschutzes in die Landnutzung verursacht mehr oder minder ausgeprägte Nutzungskonflikte. Im vorliegenden Buch wurden zur Darstellung, Einordnung und Bewertung dieser Nutzungskonflikte verschiedene, auch nicht-marktfähige Ökosystemdienstleistungen der Landnutzung wie die Erholungsfunktion von Landschaft auf der Basis einer umfassenden Bevölkerungsbefragung mit einem Choice-Experiment monetarisiert.

\section{1 Änderung von Rahmenbedingungen nach Abschluss der Studie}

\section{Horst Gömann}

Die Projektarbeiten wurden bis zum April 2016 abgeschlossen. Im Rahmen des internen Reviewprozesses für das vorliegende Buch konnten einige Anregungen zur Modifizierung und Darstellung von Modellsimulationen aufgenommen und umgesetzt werden. Eine umfangreiche Aktualisierung der Datengrundlagen erfolgte nicht, sodass die Analysen auf den zum Zeitpunkt des Projektabschlusses aktuell verfügbaren Daten basieren. Die Ergebnisse der Studie, die nicht an Gültigkeit und Aussagekraft verloren haben, werden mit Blick auf die Ableitung von Handlungsempfehlungen in den Kontext der seitdem geänderten Rahmenbedingungen eingeordnet und bewertet. Hierbei sind folgende ausgewählte Änderungen von Bedeutung: 
1. Der Preis für $\mathrm{CO}_{2}$-Emissionszertifikate im EU-Emissionshandelssystem (EU-ETS) schwankte zwischen 2013 und Mitte 2017 um $5 €$ je t $\mathrm{CO}_{2}$. Seitdem haben sich die Preise jedoch etwa verfünffacht; im April 2019 betrugen sie durchschnittlich $26 €$ je $t$ $\mathrm{CO}_{2}{ }^{1}$. Das im Jahr 2005 in Betrieb genommene EU-ETS, in dem Stromerzeuger und energieintensive Industriebetriebe $\mathrm{CO}_{2}$-Emissionszertifikate handeln können, war seit 2009 durch einen Überschuss an Emissionszertifikaten gekennzeichnet, sodass die Zertifikatpreise nahe 0 lagen. $^{2}$ Die EU-Kommission hat verschiedene Maßnahmen eingeleitet, um den Zertifikatüberschuss abzubauen. Unter anderem wurde ab Anfang 2018 eine Marktstabilitätsreserve aufgebaut. Zwischen 2019 und 2023 soll sich die Menge der in die Reserve eingestellten Zertifikate auf $24 \%$ der in Umlauf befindlichen Zertifikate verdoppeln. Ab 2021 wird die Gesamtzahl der Emissionszertifikate um 2,2 \% anstatt wie bisher um 1,7 \% jährlich gesenkt. ${ }^{3}$ Es ist zu erwarten, dass die Maßnahmen zu einem weiteren Anstieg der Zertifikatpreise führen, sodass die THG-Vermeidungskosten in diesem Sektor mit dem größten Einsparpotenzial (BMUB 2016) als Referenzgröße (Benchmark) dienen können.

2. Die EU-Mitgliedstaaten haben vereinbart, dass die Wirtschaftssektoren, die nicht unter das EU-ETS fallen - wie Verkehr, Gebäude und Landwirtschaft ihre Emissionen als Beitrag zum Gesamtziel bis 2030 um $30 \%$ gegenüber 2005 reduzieren müssen. In der im Mai 2018 angenommenen Lastenteilungsverordnung (EU 2018) wurden für alle Mitgliedstaaten verbindliche Jahresziele für die Reduzierung der THG-Emissionen im Zeitraum 2021-2030 festgelegt. Demnach müssen in Deutschland die betroffenen Sektoren ihre THG-Emissionen um $38 \%$ mindern. Sofern es zur Erreichung der Minderungsziele für Nicht-EU-ETS-Sektoren wie der Landwirtschaft notwendig ist, darf in beschränktem Umfang auf THGEmissionsgutschriften aus dem LULUCF-Sektor zurückgegriffen oder nicht benötigte Emissionsrechte in anderen Mitgliedstaaten zugekauft werden. Alle fünf Jahre erfolgt eine umfassende Überprüfung der Emissionsberichte der Mitgliedstaaten durch die EU-Kommission. Mitgliedstaaten, die keine ausreichenden Fortschritte machen, müssen geeignete Maßnahmenpläne zur Erreichung der Minderungsziele vorlegen.

3. Das in der EU-Lastenteilungsverordnung 2018/842 festgelegte Minderungsziel für Deutschland hat die Bundesregierung auf die Nicht-ETS-Sektoren in Deutschland im Rahmen des Klimaschutzplans 2050 (BMUB 2016) heruntergebrochen. Die zugeordneten Minderungsziele sollen von den Sektoren eigenverantwortlich bis 2030 erfüllt werden, wozu jeweils unterschiedliche Maßnahmen vorgeschlagen wurden. Die im Übereinkommen von Paris geforderte THG-Neutralität wird es jedoch

\footnotetext{
${ }^{1}$ https://www.eex.com/en/market-data/environmental-markets/spot-market/european-emissionallowances

${ }^{2}$ https://ec.europa.eu/clima/policies/ets_de

${ }^{3}$ https://ec.europa.eu/clima/policies/ets_de
} 
zukünftig erforderlich machen, die Gestaltungsoptionen auch in diesen Sektoren in den Klimaschutzplan einzubeziehen (BMUB 2016).

4. Aufgrund der Klage der EU gegen die Bundesrepublik Deutschland hinsichtlich des Verstoßes gegen die Nitrat-Richtlinie (Richtlinie 91/676/EG des Rates) wurde das Düngerecht novelliert (Europäische Kommission 2018, 2019). Die Novellierung umfasst Änderungen im Düngegesetz, in der Düngeverordnung sowie die Ermächtigungsgrundlage für die Bilanzierung von Stoffströmen. Im Wesentlichen zielen die Änderungen auf eine bedarfsorientierte Düngung und die Einhaltung von N-Düngungsobergrenzen (Einbeziehung von Gärresten, Klärschlamm, Kompost in die $170 \mathrm{~kg} \mathrm{~N} \mathrm{org}_{\text {org }} /$ ha - Obergrenze) (Bundesanstalt für Landwirtschaft und Ernährung (BLE) und Bundesinformationszentrum Landwirtschaft 2018). Neben dem Ziel der Reduktion von Nitratemissionen aus der Landwirtschaft in Gewässer kann erwartet werden, dass diese Maßnahmen auch zur Reduktion von Lachgasemissionen führen werden.

\subsection{Ergebnisse für den Sektor Siedlung und Verkehr}

\section{Horst Gömann}

In Deutschland wurde die Siedlungs- und Verkehrsfläche im Zeitraum von 1992 bis 2005 um rund 575.000 ha auf 4,6 Mio. ha kontinuierlich ausgedehnt. Die tägliche Flächenneuinanspruchnahme betrug in diesem Zeitraum im Mittel ca. 120 ha. Von 2005 bis 2015 wurde sie auf rund 65 ha pro Tag nahezu halbiert, sodass die Siedlungs- und Verkehrsfläche in diesem Zeitraum um etwa 300.000 ha auf 4,9 Mio. ha zunahm. Bei einer Fortschreibung der derzeitigen bzw. absehbaren gesellschaftlichen, politischen und wirtschaftlichen Rahmenbedingungen wird nach den Modellprojektionen bis zum Jahr 2030 eine Zunahme um weitere ca. 200.000 ha auf rund 5,1 Mio. ha erwartet. Dabei ist von einer Abnahme der Flächenneuinanspruchnahme auf etwa 45 ha pro Tag zum Ende des Projektionszeitraumes auszugehen (vgl. Abschn. 4.4). Trotz dieser sich bereits abzeichnenden sowie projizierten Abnahme der Flächenneuinanspruchnahme sind demnach zusätzliche Anstrengungen erforderlich, um das ,30-ha-Ziel“ der Bundesregierung zu erreichen. Darüber hinaus wird bei einer Fortschreibung der derzeitigen Rahmenbedingungen ein kontinuierlicher Flächenzuwachs des Waldes von insgesamt knapp $3 \%$ bis 2030 angenommen, wodurch sich die Waldfläche bis 2030 von 30 auf $31 \%$ erhöhen würde.

In der Studie wurde untersucht, in welchem Umfang und mit welchen Maßnahmen sich die Neuinanspruchnahme von Flächen für Siedlung, Verkehr und Erholung reduzieren lässt und wie viel Fläche für produktive Zwecke in der Land- und Forstwirtschaft verbleibt. Nach den Ergebnissen der Wirkungsanalysen ließe sich durch eine konsequente Umsetzung von Maßnahmen, die dem Klimaschutz dienen - wie der Stärkung der Innenentwicklung, der Ausschöpfung baulicher Dichte im Neubau, der 
Reduktion der Flächeninanspruchnahme durch Verkehr, der Stärkung des ÖPNV oder die zusätzliche Ausweisung von Vorrang- und Vorbehaltsgebieten -, die Flächenneuinanspruchnahme auf 20 bis 30 ha pro Tag begrenzen. Die Maßnahmen führen ferner $\mathrm{zu}$ einer besseren verkehrlichen Erreichbarkeit. Dies unterstützt eine Veränderung des Modal Split und kann den hohen Anteil des motorisierten Individualverkehrs und damit beträchtliche THG-Emissionen reduzieren. Im langfristig angelegten Umbau der Siedlungs-, Gebäude- und Verkehrsinfrastruktur liegt das größte THG-Einsparpotenzial, für das im Rahmen der Studie keine Vermeidungskosten beziffert werden konnten.

Auch wenn deutschlandweit betrachtet in der Klimaschutzstrategie die Flächeninanspruchnahme deutlich reduziert werden kann, ist davon auszugehen, dass es weiterhin Regionen mit hohem Flächenzuwachs gibt. Von einem weiterhin hohen Flächenzuwachs trotz der durchgeführten Maßnahmen wird im Umland der Großstädte (v. a. Hamburg, Berlin, München), im Rheinland, der Metropolregion Rhein-Neckar und im westlichen Niedersachsen (Emsland) auszugehen sein. In weiten Landesteilen Sachsens, SachsenAnhalts und Mecklenburg-Vorpommerns könnte aufgrund des hohen Leerstands ein Netto-Rückbau stattfinden, der entsprechend begleitet werden muss, um die bestehenden Strukturen zu stärken. In vielen kreisfreien Städten könnte die Baulandnachfrage vollständig durch Innenentwicklung befriedigt werden. Hierzu zählen viele Städte im Ruhrgebiet (z. B. Essen, Dortmund, Herne), von denen einige aufgrund des hohen Leerstands auch Netto-Rückbau betreiben könnten (z. B. Gelsenkirchen, Bochum), aber auch andere kreisfreie Städte - vor allem solche mit Bevölkerungsrückgang - könnten ihre Außenentwicklung durch Maßnahmen der Innenentwicklung, Konzentration und Entsiegelung drastisch reduzieren, wie z. B. Offenbach, Ludwigshafen, Wuppertal, Solingen, Krefeld oder Kassel.

Eine vergleichbar hohe Verringerung der täglichen Flächeninanspruchnahme ließe sich durch ein ähnliches, allerdings auf Natur- und Umweltschutz ausgerichtetes Maßnahmenbündel erzielen, wobei der restriktivere Freiraumschutz eine hohe Wirkung erzielt. Hinsichtlich des Flächensparens sind die Maßnahmen der Natur- und Umweltschutzstrategie zwar weniger restriktiv, bewirken aber dennoch eine erkennbare Verbesserung beim Erhalt von aus naturschutzfachlicher Sicht schützenswerten Räumen.

Wird ein stärkerer Fokus auf Klimaanpassung gelegt, ginge das aufgrund der dafür notwendigen Flächenerfordernisse zu Lasten einer Verringerung der Flächenneuinanspruchnahme, die sich nur auf 40 ha pro Tag reduzieren ließe. Wichtige flächenrelevante Maßnahmen sind hierbei der Rückzug aus der Fläche (dezentrale Konzentration), Erhalt und Entwicklung innerstädtischer Freiflächen, zusätzliche Ausweisung von Vorrangund Vorbehaltsgebieten und die Stärkung des Hochwasserschutzes. Eine Auflockerung der Bebauung, die Schaffung, Sicherung und klimaeffiziente Bewirtschaftung klimarelevanter Grünflächen sowie die Erhaltung, strukturelle Verbesserung und Schaffung von Luftleitbahnen (insbesondere linearer Grün- und Wasserflächen) reduzieren den innerstädtischen Hitzeinseleffekt. 


\section{Raum-, Regional- und Bauleitplanung stärken}

Für ein nachhaltiges Landnutzungsmanagement ist vor dem Hintergrund der komplexen Wechselwirkungen und Zielkonflikte zwischen und innerhalb der Landnutzungssektoren darauf hinzuwirken, verstärkt integrierte und koordinierende Planungs- und Handlungsansätze zur Anwendung zu bringen. Besondere Bedeutung kommt hier der räumlichen Gesamtplanung zu. Diese kann im Rahmen der Raumordnung sowie der Kommunalplanung dazu beitragen, die im Zuge des Klimawandels und der Energiewende zunehmenden Flächennutzungskonflikte frühzeitig $\mathrm{zu}$ erkennen und koordinierend auf diese einzuwirken. Zudem können auf Ebene der Landes- und Regionalplanung Klimaschutzmaßnahmen sowie Maßnahmen zur Klimaanpassung vorbereitet und entsprechende Flächen gesichert werden. Insbesondere die Raumordnung ist geeignet, entsprechende Risikovorsorge durch die Ausweisung von Vorrang- und Vorbehaltsgebieten und die Steuerung der Siedlungsentwicklung zu leisten.

\section{Klimaschutzabgabe für die Flächenneuinanspruchnahme einführen}

Die Neuinanspruchnahme von Flächen durch Siedlung und Verkehr erfolgt vor allem zu Lasten der landwirtschaftlich genutzten Fläche, sodass in Deutschland das Produktionspotenzial für Nahrungs- und Futtermittel sowie Biomasse kontinuierlich abnimmt. Infolgedessen nehmen bei unterstelltem unverändertem Konsumverhalten die Importe zu, die andernorts zu einer Erhöhung von THG-Emissionen (iLUC-Effekt) führen, es sei denn, der Rückgang der landwirtschaftlich genutzten Fläche in Deutschland wird durch Steigerungen der Flächenproduktivität überkompensiert. Nach den Modellergebnissen ließen sich durch eine konsequente Umsetzung flächensparender Maßnahmen mit positiven Wirkungen sowohl auf den Klimaschutz als auch den Natur- und Umweltschutz bis zu 200.000 ha Flächenneuinanspruchnahme vermeiden, die dem THGBilanzierungssektor Landnutzung, Landnutzungsänderungen und Forstwirtschaft (LULUCF) zuzurechnen sind.

Die direkten THG-Emissionen der Landnutzungsumwandlung belaufen sich laut UBA (2018) gemessen am impliziten Emissionsfaktor für die Landnutzungsänderung zur Kategorie Siedlungen in Deutschland im Durchschnitt auf 2,6 t CO $\mathrm{CO}_{2}$ Äqu./ha (siehe auch WBAE und WBW 2016). Dem sind iLUC-Effekte in Höhe von jährlich $7 \mathrm{t} \mathrm{CO}_{2}$ - ̈̈qu. je ha hinzuzurechnen. Das THG-Vermeidungspotenzial beläuft sich somit auf insgesamt rund 1,8 Mio. $\mathrm{C} \mathrm{CO}_{2}$ - ̈̈qu. pro Jahr (siehe auch Abschn. 4.5).

Im Bereich Siedlung und Verkehr gibt es derzeit kein Instrument, das den THGEffekt der Neuinanspruchnahme von Flächen bepreist. Angesichts der oben genannten Rahmenbedingungen, insbesondere der EU-Lastenteilungsverordnung, ist eine stärkere Berücksichtigung der nicht marktfähigen Ökosystemleistung Klimaschutz anzustreben. Aus diesem Grund wird die Etablierung eines Fonds zur Finanzierung von Klimaschutzprojekten, etwa in Anlehnung an die Funktionsweise des Waldklimafonds, angeregt. Die Erhebung einer Klimaschutzabgabe auf die Flächenneuinanspruchnahme ist eine konsequente Klimaschutzmaßnahme, auch wenn ihre lenkende Wirkung auf die Inanspruchnahme von Flächen vernachlässigbar gering ausfällt. 


\subsection{Ergebnisse und Empfehlungen für die Landwirtschaft}

Horst Gömann, Johanna Fick und Martin Henseler

Während die Landwirtschaftsfläche laut Liegenschaftskataster im Zeitraum von 1990 bis 2014 um 1,0 Mio. ha auf 18,5 Mio. ha zurückging, nahm die von landwirtschaftlichen Betrieben bewirtschaftete landwirtschaftlich genutzte Fläche (LF) lediglich um 0,5 Mio. ha auf 16,7 Mio. ha ab. Rund die Hälfte der Inanspruchnahme von Flächen für Siedlung und Verkehr sowie für den Ausgleich der dadurch bedingten Eingriffe in die Natur entfiel in der Summe auf Landwirtschaftsflächen, die nicht von landwirtschaftlichen Betrieben bewirtschaftet wurden. Über die tatsächliche Nutzung dieser Flächen liegen keine statistischen Informationen vor. Ihr Umfang reduzierte sich im genannten Zeitraum von 2,3 auf 1,8 Mio. ha.

Bis zum Jahr 2030 wird ein weiterer Rückgang der LF auf 16,5 Mio. ha projiziert. Unter Berücksichtigung der erwarteten Produktivitätsentwicklungen, Faktoreinsatzmengen und ökonomischen Rahmenbedingungen werden den Modellergebnissen zufolge die Anbauflächen von Weizen, Raps und Mais zu Lasten anderer Kulturen ausgedehnt. Es wird erwartet, dass der Viehbestand in Deutschland bei ca. 8 Mio. Großvieheinheiten (GVE) nahezu konstant bleibt, mit leichten Verschiebungen zugunsten von Schweinen und Geflügel.

Die THG-Emissionen in der Landwirtschaft verringerten sich im Zeitraum von 1990 bis 2014 um $18 \%$ auf 72 Mio. t CO -Äqu. (BMUB 2016, S. 33), hauptsächlich infolge des Viehbestandsabbaus Anfang der 1990er-Jahre in den fünf östlichen Bundesländern. Nach der im RAUMIS-Modell vorgenommenen Systemabgrenzung, die von der Systematik in der nationalen THG-Berichterstattung abweicht, beliefen sich die THGEmissionen im Basisjahr 2010 auf rund 73 Mio. t $\mathrm{CO}_{2}$ - ̈̈qu. Im Vergleich dazu weisen WBAE und WBW (2016) für das Jahr 2010 insgesamt 62,3 Mio. t $\mathrm{CO}_{2}$ 24,6 Mio. t $\mathrm{CO}_{2}$-Äqu. (39\%) für Fermentation $\left(\mathrm{CH}_{4}\right)$, 10,2 Mio. t $\mathrm{CO}_{2}$-Äqu. (16 \%) für Düngerwirtschaft $\left(\mathrm{N}_{2} \mathrm{O}\right.$ und $\left.\mathrm{CH}_{4}\right), 24,1$ Mio. t $\mathrm{CO}_{2}$ - $\mathrm{Äqu}$. (39\%) für Landwirtschaftliche Böden $\left(\mathrm{N}_{2} \mathrm{O}\right), 3,4$ Mio. t $\mathrm{CO}_{2}$ - ̈̈qu. (5 \%) für andere $\mathrm{CH}_{4}^{-}, \mathrm{N}_{2} \mathrm{O}$ - und $\mathrm{CO}_{2}$-Emissionen (z. B. Kalkung, Harnstoff) (WBAE und WBW 2016, S. 19, Tab. 2.1)

Angesichts der unterstellten landwirtschaftlichen Produktionsentwicklungen bleiben die THG-Emissionen der Landwirtschaft nach den Modellergebnissen bis zum Jahr 2030 weitgehend konstant. Diese Projektionen decken sich mit den erwarteten Entwicklungen in der Thünen-Baseline 2018 (Offermann et al. 2018).

Die in der Studie hinsichtlich ihres THG-Einsparungspotenzials untersuchten wichtigsten Bereiche der landwirtschaftlichen Landnutzung sind die Nutzung organischer Böden, die Anpassung des Düngemanagements, die Substitution fossiler Energie durch Biomasse sowie die Erhaltung und Schaffung von THG-Senken. Der ebenfalls wichtige Bereich der tierhaltungsbedingten THG-Emissionen wurde im Rahmen der Studie nicht betrachtet, weil sie nur einen indirekten Einfluss über den Futterbedarf und die Nährstoffeffizienz von Wirtschaftsdüngern auf die landwirtschaftliche Landnutzung haben. Gleichwohl ist die Tierproduktion ein wesentlicher Bestandteil 
des Gesamtsystems Landwirtschaft und ist bei der Ableitung zu empfehlender Handlungsoptionen für einen verstärkten Beitrag der Landwirtschaft zum Klimaschutz zu berücksichtigen.

Im Klimaschutzplan 2050 der Bundesregierung ist für die Landwirtschaft eine THG-Emissionsminderung auf 58-61 Mio. t $\mathrm{CO}_{2}$-Äqu., d. h. um weitere rund $17 \%$ gegenüber dem Jahr 2014, avisiert (BMUB 2016, S. 33). Nach den Wirkungsanalysen in dieser Studie wäre dieses Ziel durch die untersuchte klimaschutzorientierte Maßnahmenkombination einschließlich der zu berücksichtigenden iLUC-Effekte erreichbar: Eine Wiedervernässung von rund 270.000 ha landwirtschaftlich genutzter organischer Böden, eine Abgabe auf mineralischen Stickstoff zur Steigerung der Düngeeffizienz sowie die Aufforstung von rund 0,5 Mio. ha landwirtschaftlicher Grenzstandorte würden nach den Modellergebnissen die THG-Emissionen um rund 11,8 Mio. $\mathrm{t}$ $\mathrm{CO}_{2}$ - ̈̈qu. verringern. Allerdings ist hierbei zu berücksichtigen, dass die anrechenbare THG-Emissionsreduktion, insbesondere der Abgabe auf mineralischen Stickstoff, geringer ausfällt als in der vorliegenden Studie, in der die Lachgas-Emissionen auf der Basis regionalisierter Faktoren ermittelt wurden, was derzeit nicht dem internationalen Standard der THG-Berichterstattung entspricht.

Eine vergleichbare Klimaschutzwirkung könnte ebenfalls mit einer bioenergieorientierten Landnutzungsstrategie erreicht werden, bei der der Energiemaisanbau gegenüber der Referenzprojektion im Jahr 2030 noch einmal um 1 Mio. ha ausgedehnt und auf den wiedervernässten organischen Böden zusätzlich Paludikulturen angebaut werden. Die Minderungswirkung beläuft sich auf rund 14,5 Mio. t $\mathrm{CO}_{2}$ - ̈̈qu. Dem stehen negative Effekte auf die Nährstoffbelastung des Grundwassers, auf das Landschaftsbild und - je nach konkreter Ausgestaltung der Anbauverfahren - gegebenenfalls auch auf die Biodiversität auf landwirtschaftlichen Flächen gegenüber und deutlich höhere Vermeidungskosten je $\mathrm{t} \mathrm{CO}_{2}$, sodass die Gesamtbewertung dieser Strategie ambivalent und damit aus gesellschaftlicher Sicht deutlich schlechter ausfällt als die anderen untersuchten Strategien.

In einer auf Natur- und Umweltschutz orientierten Landnutzungstrategie wurden die Auswirkungen einer Extensivierung von insgesamt $10 \%$ der landwirtschaftlich genutzten Fläche einschließlich 270.000 ha landwirtschaftlich genutzter organischer Böden analysiert. Den mit der Extensivierung vergleichsweise geringen THGMinderungen stehen hohe iLUC-Effekte gegenüber, sodass das in der Natur- und Umweltschutzstrategie definierte Maßnahmenbündel nur bedingt geeignet ist, die Klimaschutzziele zu erreichen.

Die Bevölkerungsbefragung ergab eine deutlich positive Bewertung für die klimaschutzfokussierte sowie die natur- und umweltschutzorientierte Strategie aus gesamtgesellschaftlicher Sicht. Die erheblichen Nutzen, die in den Dimensionen Klima- und Gewässerschutz, Landschaftsbild sowie Erhaltung und Erhöhung der Biodiversität erzielt werden können, übertreffen die jeweiligen betriebswirtschaftlichen Einbußen deutlich. Würde die umwelt- und naturschutzorientierte Strategie kombiniert mit einer Vermehrung von Waldflächen und weiteren Klimaschutzmaßnahmen, die mit Natur- 
schutzzielen gut zu verbinden wären (beispielsweise den Anbau von Paludikulturen auf wiedervernässten Moorflächen), ergäbe sich ein noch größerer positiver volkswirtschaftlicher Gesamteffekt - dabei wäre eine vollständige Kompensation der damit verbundenen betriebswirtschaftlichen Einbußen der landwirtschaftlichen Betriebe bereits berücksichtigt.

Aus den modellgestützten Ergebnissen lassen sich folgende Empfehlungen für klimaschutzorientierte Handlungsoptionen in der Landwirtschaft ableiten:

\section{Landwirtschaftlich genutzte Moore standortspezifisch schützen}

Auf die landwirtschaftliche Nutzung organischer Böden (Moore) entfällt ein beträchtlicher Anteil der durch die Landwirtschaft verursachten THG-Emissionen. Aufgrund des großen Potenzials auf einer vergleichsweise geringen Fläche sollten jeweils standortspezifisch Optionen geprüft werden, THG-Emissionen durch eine Anpassung der landwirtschaftlichen Flächennutzung zu mindern. Maßnahmen zum Moorschutz können in der Regel nicht von einzelnen landwirtschaftlichen Betrieben umgesetzt werden, da beispielsweise die Wiedervernässung von Mooren großflächige Veränderungen der Landnutzung in einem bestimmten Gebiet erfordert. Daraus ergibt sich die Notwendigkeit einer planerischen Begleitung derartiger Vorhaben und des Einsatzes eines gut aufeinander abgestimmten Maßnahmen- und Instrumentenbündels im regionalen und lokalen Kontext. Einzelne Schritte und Handlungsoptionen für einen standortspezifischen Moorschutz haben der WBAE und WBW in ihrem Gutachten (2016) ausführlich dargelegt.

Ein Instrument zur Finanzierung von Maßnahmen zur Wiedervernässung sind seit 2012 so genannte MoorFutures. MoorFutures sind Kohlenstoffzertifikate, die sich an internationalen Standards wie beispielsweise dem Verified Carbon Standard orientieren und für den freiwilligen Kohlenstoffmarkt entwickelt wurden. Bisher wird der freiwillige Markt von Klimaschutzprojekten aus den Bereichen Erneuerbare Energien, der Energieeffizienz sowie Aufforstung bestimmt. Ein MoorFuture entspricht der Emissionsminderung von einer Tonne $\mathrm{CO}_{2}$ - ̈̈quivalente. MoorFutures werden aus regionalen Projekten zur Moorwiedervernässung erzeugt und sind damit eindeutig auf konkrete regionale Projekte inklusive einer freizugänglichen Dokumentation zurückzuführen. Anwendung finden MoorFutures bisher bei der Projektfinanzierung von Moorwiedervernässungen in Mecklenburg-Vorpommern, Brandenburg und SchleswigHolstein (Ministerium für Landwirtschaft und Umwelt Mecklenburg-Vorpommern 2017).

\section{Strukturen und Anreize zur Steigerung der Stickstoffeffizienz etablieren}

Verbesserungen bei der Ausnutzung von Stickstoff in der Landwirtschaft weisen ein weiteres bedeutsames Potenzial auf, Emissionen THG-relevanter Stickstoffverbindungen zu vermeiden. Diesbezüglich weist der Klimaschutz Parallelen und gemeinsame Ziele zum Wasserschutz auf. Durch die Steigerung der N-Effizienz mit den dargelegten Maßnahmen kann der N-Einsatz in der Landwirtschaft deutlich gesenkt werden. Die damit einhergehende Minderung der THG-Emissionen wird dem landwirtschaftlichen Sektor angerechnet. Die im Bereich des landwirtschaftlichen Gewässerschutzes 
geltenden gesetzlichen Rahmenbedingungen sollten wie folgt zu einem umfassenderen System weiterentwickelt werden, in dem Anreize zur Steigerung der N-Effizienz in der Landwirtschaft implementiert werden und ihre volle Wirkung entfalten können.

\section{Weiterentwicklung der Düngegesetzgebung}

Die EU-Nitratrichtlinie (EU 1991) zur Vermeidung des Eintrags von Nitrat in Gewässer aus landwirtschaftlichen Quellen wird in Deutschland insbesondere durch die Düngeverordnung (DüV) umgesetzt. Die EU-Kommission hat im Jahr 2016 gegen Deutschland ein Vertragsverletzungsverfahren eingeleitet wegen unzureichender Umsetzung der Nitratrichtlinie. In diesem Zusammenhang traten in Deutschland nach einer Änderung des Düngegesetzes im April 2017 eine novellierte Düngeverordnung im Juni 2017 und eine Stoffstrombilanzverordnung (StoffBilV) im Januar 2018 in Kraft. In der novellierten DüV wurden Kontrollwerte für die Düngung mit organischen Stickstoffdüngern (z. B. Wirtschaftsdünger und Gärreste pflanzlichen Ursprungs) sowie maximale N-Überschüsse verschärft. Eine indirekte Verschärfung resultiert aus der Absenkung einiger Pauschalwerte für N-Verluste, beispielsweise im Stall oder bei der Lagerung und Ausbringung von organischen Düngern, die gemäß DüV bei der Bilanzierung des betrieblichen N-Überschusses (Feld-Stall-Bilanz) abgezogen werden können. Zusätzlich sind landwirtschaftliche Betriebe ab einem gewissen Viehbestand verpflichtet, eine Stoffstrombilanz (Hoftorbilanz) gemäß StoffBilV zu erstellen.

Die Vorgaben der DüV (2017) lassen einen Rückgang der N-Überschüsse erwarten, was zugleich eine gewisse Minderung der THG-Emissionen bewirkt. Darüber hinaus bestehen in einem landwirtschaftlichen Betrieb mit Blick auf den Klimaschutz kaum Anreize, stickstoffbürtige THG-Emissionen zu verringern, sofern er die gesetzlichen Standards der DüV einhält.

Daher sollten die nach DüV und StoffBilV vorgeschriebene Feld-Stall- bzw. Hoftorbilanz zu einem betrieblichen Nährstoffmanagementsystem zusammengeführt werden, nicht zuletzt, um Probleme - vor allem im Vollzug - zu vermeiden, die die unterschiedlichen Ergebnisse der beiden Bewertungssysteme mit sich bringen. Wie bei der Stoffstrombilanz sollten sämtliche Nährstoffimporte (z. B. in Futter- und Düngemitteln) den Nährstoffexporten (z. B. in pflanzlichen und tierischen Produkten, aber auch Wirtschaftsdüngern) ohne Berücksichtigung pauschaler Verluste gegenübergestellt werden. In die erforderliche Dokumentationspflicht der landwirtschaftlichen Betriebe ${ }^{4}$ über die Stoffströme sollte der vor- und nachgelagerte Bereich der Landwirtschaft (z. B. Landhandel)

\footnotetext{
${ }^{4}$ Betriebe, die überregional transportierte Wirtschaftsdünger aufnehmen, müssen dies gemäß der Verordnung über das Inverkehrbringen und Befördern von Wirtschaftsdünger (WDüngV) in einer Datenbank melden. In einigen Bundesländern bestehen zusätzlich Landesverordnungen, die wirtschaftsdüngerabgebende Betriebe verpflichten, die an andere Betriebe abgegebenen Nährstoffmengen in einer zentralen Datenbank zu melden.
} 
wie in Dänemark und den Niederlanden ${ }^{5}$ einbezogen werden. Einzuhaltende Kontrollwerte (Mindeststandards) sind so festzulegen, dass sie den Vorgaben der derzeitigen Düngegesetzgebung entsprechen. Überschreitungen der Kontrollwerte sollten als „Cross Compliance“-relevante Verstöße im Rahmen eines Ordnungswidrigkeitsverfahrens geahndet werden, was mit einer Abgabe auf den N-Überschuss vergleichbar ist. Um Ziele des Klima- bzw. Gewässerschutzes zu erreichen, können die Kontrollwerte kontinuierlich gesenkt werden. Bezüglich der detaillierten, praxistauglichen Ausgestaltung eines umfassenden Nährstoffmanagementsystems besteht Forschungsbedarf.

Im Rahmen des Nährstoffmanagementsystems können landwirtschaftliche Betriebe die erforderlichen N-Effizienzverbesserungen betriebsspezifisch erzielen, beispielsweise durch Anpassung der Fütterung, Investitionen in Wirtschaftsdüngerlager und deren Abdeckung ${ }^{6}$ sowie den Einsatz von Nitrifikationshemmern oder bodennahe, sensorgestützte Ausbringungstechnologien für Wirtschaftsdünger. In den letzten Jahren wurden Investitionen in derartige Technologien in einigen Bundesländern durch Fördermaßnahmen flankiert. Die daraus resultierenden N-Effizienzverbesserungen lassen sich im derzeit bestehenden System jedoch nur unzureichend darstellen. Um Anreize zur Minderung des N-Einsatzes über den gesetzlichen Standard hinaus zu schaffen, sollte eine Förderung durch eine Agrarumwelt- und Klimamaßnahme in Erwägung gezogen werden.

\section{Abgabe auf mineralischen Stickstoffdünger}

Die Wirkungen einer Verteuerung des Einsatzes mineralischer Stickstoffdüngemittel durch eine Abgabe lassen sich durch die Modellanalysen größenordnungsmäßig abschätzen. Das in der vorliegenden Studie verwendete RAUMIS-Modell simuliert die Düngung auf der Basis flexibel kalibrierbarer Produktionsfunktionen, die aus Düngungsversuchen abgeleitet wurden (Weingarten 1996; vgl. Abschn. 4.5.2.2). Die Verteuerung des Einsatzes mineralischen Stickstoffs um $40 \%$ führt nach den Modellergebnissen zu einem Rückgang der Einsatzmenge um $12 \%$. Aufgrund der geringen Eigenpreiselastizität (rund -0,3) wäre eine deutlich höhere Abgabe auf mineralischen Stickstoff erforderlich, um substanzielle Reduktionen seines Einsatzes zu erzielen. Das würde in erster Linie Betriebe in Ackerbauregionen betreffen, die den verteuerten Mineralstickstoff nicht durch eine effizientere Nutzung von Wirtschaftsdünger, der in den Regionen in geringem Umfang anfällt, substituieren können.

\footnotetext{
${ }^{5}$ In den Niederlanden müssen Landhändler, landwirtschaftliche Betriebe sowie Verarbeitungsunternehmen in die zentrale Datenbank „Kringloopwijzer“ melden.

${ }^{6}$ Lagerkapazitäten müssen die Anforderungen an Jauche-, Gülle- und Silagesickersaftanlagen der Anlage 7 der Verordnung über Anlagen zum Umgang mit wassergefährdenden Stoffen (AwSV) genügen, die im August 2017 in Kraft trat.
} 
Das Düngungsverhalten wird in der Praxis durch weitere Faktoren determiniert, vor allem durch risikoaverses Verhalten der landwirtschaftlichen Betriebe (Isermeyer 1992). Angesichts der stark vom Witterungsverlauf abhängigen Pflanzenverfügbarkeit des in Wirtschaftsdüngern gebundenen Stickstoffs erfolgen zum Teil beträchtliche Sicherheitsaufschläge mit mineralischem Stickstoff, auch und gerade in Betrieben mit hohem Wirtschaftsdüngeranfall (LWK NRW 2017). Weitere Ursachen für einen zu hohen mineralischen Stickstoffeinsatz dürften in einer Überschätzung des Ertragspotenzials sowie in einer Unterschätzung der mit Hilfe bodennaher Ausbringungstechniken deutlich höheren Wirksamkeit und Pflanzenverfügbarkeit organischer Dünger liegen.

Vor diesem Hintergrund ist die sukzessive Einführung einer moderaten Abgabe bis $40 \%$ auf den Einsatz mineralischen Stickstoffs eine sinnvolle Maßnahme. So haben sich auch WBAE und WbW (2016) für die Einführung einer Mineralstickstoffabgabe ausgesprochen, wenn die Verschärfung des Düngerechts bis 2020 keine hinreichende Verringerung der Nitratüberschüsse bewirkt, um die oben dargelegte Weiterentwicklung der Düngegesetzgebung zu ergänzen. Viele Betriebe würden zunächst den Sicherheitsaufschlag neu bewerten, dessen Verringerung keine signifikanten Ertragseinbußen erwarten lässt. Ferner steigt durch die Mineralstickstoffabgabe gleichzeitig der Wert des im Wirtschaftsdünger enthaltenen Stickstoffs, sodass ein höherer Anreiz besteht, diesen effizienter einzusetzen. Die durch die Abgabe vereinnahmten Mittel könnten eingesetzt werden, um sie als Direktzahlungsaufschlag produktionsneutral an die Landwirtschaft zu erstatten, gezielt zur Förderung N-effizienter Technologien einzusetzen oder Betriebe zu honorieren, deren Nährstoffüberschüsse (deutlich) unterhalb der gesetzlichen Standards liegen.

\section{Entwicklung einer zertifizierungsfähigen, betrieblichen THG-Bilanzierung}

Tatsächliche landnutzungsbedingte THG-Emissionen sind im Sektor Landwirtschaft aufgrund der vielfältigen, von natürlichen Faktoren abhängigen Produktionsprozesse in der Praxis nicht quantifizierbar. Aus diesem Grund werden in der nationalen THGInventarberechnung für die Landwirtschaft nur die in den einschlägigen internationalen Regelwerken vorgegebenen, teilweise sehr einfachen Methoden verwendet (Haenel et al. 2018). Darüber hinaus liegen auf sektoraler Ebene für viele THG-Emissionsquellen keine belastbaren Daten vor. Beispielsweise wird in der nationalen Inventarberechnung die Annahme der bedarfsgerechten Tierfütterung getroffen (Haenel et al. 2018). Anpassungen der tatsächlichen Fütterung, einschließlich der Verringerung von Futterverlusten, wirken sich somit nicht auf die nationalen THG-Emissionen des Sektors Landwirtschaft aus.

Anders als für Unternehmen, die am EU-ETS teilnehmen, existiert für landwirtschaftliche Betriebe derzeit kein systematischer Rahmen, der zur Honorierung der Umsetzung betriebsindividueller Klimaschutzmaßnahmen genutzt werden könnte. Aus diesem Grund sollte eine zertifizierungsfähige Methodik zur THG-Bilanzierung landwirtschaftlicher Betriebe entwickelt werden. Als eine Grundlage hierfür kann der von einer bundesweiten Arbeitsgruppe entwickelte Berechnungsstandard für einzelbetrieb- 
liche Klimabilanzen (BEK) in der Landwirtschaft verwendet werden (Effenberger et al. 2016). Ein großer Teil der für eine einzelbetriebliche THG-Bilanzierung erforderlichen Informationen und Daten ist im Rahmen der Düngegesetzgebung zu dokumentieren. Der Schwerpunkt der einzelbetrieblichen Bewertung liegt weniger auf den absoluten Veränderungen der THG-Emissionen, sondern auf den THG-Emissionen je Produkteinheit.

Die der Landwirtschaft nachgelagerten Unternehmen der Ernährungsindustrie sowie des Groß- und Einzelhandels haben zunehmend höhere Anforderungen an die Nachhaltigkeit der landwirtschaftlichen Produktion. Die Auflagen reichen beispielsweise von deutlich unter den gesetzlichen Grenzwerten festgelegten Höchstrückstandsmengen von Pflanzenschutzmitteln in den Produkten bis hin zur Etablierung der Berechnung von $\mathrm{CO}_{2}$-Fußabdrücken je Produkteinheit. In der Regel wird das Erreichen definierter Nachhaltigkeitsziele durch Anreizkomponenten gefördert bzw. das Nichteinhalten festgelegter Standardqualitäten durch Preisabschläge sanktioniert. Die steigenden Anforderungen von Unternehmen des nachgelagerten Bereichs erfordern von den landwirtschaftlichen Betrieben zunehmende Dokumentationen. Es sollte geprüft werden, inwiefern eine einzelbetriebliche THG-Bilanzierung auch im Rahmen von AUKM genutzt und mit einer Anreizkomponente für klimaschützendes Verhalten gekoppelt werden kann. Auf diese Weise könnte die Klimaschutzwirkung vieler Natur- und Umweltschutzmaßnahmen mit bewertet und honoriert werden.

\section{Literatur}

Bundesministerium für Umwelt, Naturschutz, Bau und Reaktorsicherheit (BMUB) (2016) Klimaschutzplan 2050 - Klimaschutzpolitische Grundsätze und Ziele der Bundesregierung. www. bmub.bund.de. Zugegriffen: 12. März 2018

Bundesanstalt für Landwirtschaft und Ernährung (BLE) und Bundesinformationszentrum Landwirtschaft (2018) Die neue Düngeverordnung. ISBN 978-3-8308-1323-1

Effenberger M, Gödeke K, Grebe S, Haenel HD, Hansen A, Häußermann U, Kätsch S, Lasar A, Nyfeler-Brunner A, Osterburg B, Paffrath P, Poddey E, Schmid H, Schraml M, Wulf S, Zerhusen B (2016) Berechnungsstandard für einzelbetriebliche Klimabilanzen (BEK) in der Landwirtschaft. Herausgeber. Kuratorium für Technik und Bauwesen in der Landwirtschaft e. V. (KTBL), Darmstadt. https://www.ktbl.de/fileadmin/user_upload/Allgemeines/Download/ BEK/Handbuch.pdf. Zugegriffen: 20. März 2019

Europäische Kommission (2019) Nitrat im Grundwasser: Kommission mahnt Deutschland zur Umsetzung des EuGH-Urteils. https://ec.europa.eu/germany/news/20190725-nitrat_de. Zugegriffen: 15. Dez. 2019

Europäische Komission (2018) Wasserverschmutzung: Kommission fordert DEUTSCHLAND zur Umsetzung des Urteils wegen Verstoßes gegen EU-Nitratvorschriften auf. https://ec.europa.eu/ commission/presscorner/detail/de/INF_19_4251 Zugegriffen: 15. Dez. 2019

EU (1991) Richtlinie 91/676/EWG des Rates vom 12. Dezember 1991 zum Schutz der Gewässer vor Verunreinigung durch Nitrat aus landwirtschaftlichen Quellen

EU (2018) Verordnung (EU) 2018/842 des Europäischen Parlaments und des Rates vom 30. Mai 2018 zur Festlegung verbindlicher nationaler Jahresziele für die Reduzierung der Treibhausgasemissionen im Zeitraum 2021 bis 2030 als Beitrag zu Klimaschutzmaßnahmen zwecks 
Erfüllung der Verpflichtungen aus dem Übereinkommen von Paris sowie zur Änderung der Verordnung (EU) Nr. 525/2013. Amtsblatt der Europäischen Union. L 156/26 vom 19.06.2018

Haenel H-D, Rösemann C, Dämmgen U, Döring U, Wulf S, Eurich-Menden B, Freibauer A, Döhler H, Schreiner C, Osterburg B (2018) Calculations of gaseous and particulate emissions from German agriculture 1990-2016: Report on methods and data (RMD) submission 2018. Thünen Rep 57:424. Braunschweig: Johann Heinrich von Thünen-Institut

Isermeyer F (1992) Optimaler Stickstoffeinsatz in der Landwirtschaft aus betriebswirtschaftlicher und volkswirtschaftlicher Sicht. Landbauforschung Völkenrode 132:5-20

Landwirtschaftskammer Nordrhein-Westfalen (LWK NRW) (2017) Nährstoffbericht 2017 über Wirtschaftsdünger und andere organische Düngemittel für Nordrhein-Westfalen. Erstellt im Auftrag des Ministeriums für Umwelt, Landwirtschaft, Natur- und Verbraucherschutz Nordrhein-Westfalen. https://www.landwirtschaftskammer.de/landwirtschaft/ackerbau/pdf/ naehrstoffbericht-2017.pdf. Zugegriffen: 20. März 2019

Ministerium für Landwirtschaft und Umwelt Mecklenburg-Vorpommern (2017) Der MoorFutures Standard. Verantwortlich: Ministerium für Landwirtschaft und Umwelt Mecklenburg-Vorpommern (Abteilung 2) und die Arbeitsgruppe MoorFutures der Länder Mecklenburg-Vorpommern, Brandenburg und Schleswig-Holstein gemeinsam mit dem Wissenschaftlichen Beirat MoorFutures. https://www.moorfutures.de/downloads/. Zugegriffen: 06. Sept. 2019

Offermann F, Banse M, Freund F, Haß M, Kreins P, Laquai V, Osterburg B, Pelikan J, Rösemann C, Salamon P (2018) Thünen-Baseline 2017-2027: Agrarökonomische Projektionen für Deutschland. Thünen Rep 56:116. Johann Heinrich von Thünen-Institut, Braunschweig

Umweltbundesamt (UBA) (2018) Berichterstattung unter der Klimarahmenkonvention der Vereinten Nationen und dem Kyoto-Protokoll 2018. Nationaler Inventarbericht zum Deutschen Treibhausgasinventar 1990-2016. Umweltbundesamt - UNFCCC-Submission

Wissenschaftlicher Beirat Agrarpolitik, Ernährung und gesundheitlicher Verbraucherschutz beim BMEL (WBAE) und Wissenschaftlicher Beirat Waldpolitik beim BMEL (WBW) (2016) Klimaschutz in der Land- und Forstwirtschaft sowie den nachgelagerten Bereichen Ernährung und Holzverwendung. Gutachten, Berlin

Open Access Dieses Kapitel wird unter der Creative Commons Namensnennung 4.0 International Lizenz (http://creativecommons.org/licenses/by/4.0/deed.de) veröffentlicht, welche die Nutzung, Vervielfältigung, Bearbeitung, Verbreitung und Wiedergabe in jeglichem Medium und Format erlaubt, sofern Sie den/die ursprünglichen Autor(en) und die Quelle ordnungsgemäß nennen, einen Link zur Creative Commons Lizenz beifügen und angeben, ob Änderungen vorgenommen wurden.

Die in diesem Kapitel enthaltenen Bilder und sonstiges Drittmaterial unterliegen ebenfalls der genannten Creative Commons Lizenz, sofern sich aus der Abbildungslegende nichts anderes ergibt. Sofern das betreffende Material nicht unter der genannten Creative Commons Lizenz steht und die betreffende Handlung nicht nach gesetzlichen Vorschriften erlaubt ist, ist für die oben aufgeführten Weiterverwendungen des Materials die Einwilligung des jeweiligen Rechteinhabers einzuholen.

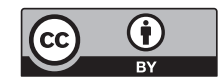

